\title{
Influencia del sistema de mínima cantidad de lubricante (MQL) en la vida de las herramientas de corte de metal duro en el fresado del acero AISI 1018
}

\section{(Influence of minimum quantity of lubricant (MQL) on tool life of carbide cutting tools during milling process of steel AISI 1018)}

\author{
Núñez Diego ${ }^{1}$, Núñez Byron ${ }^{1}$, Vaca Henry ${ }^{1}$, Ureña Maritza ${ }^{1}$
}

\begin{abstract}
Resumen:
Hoy en día, la alta productividad del mecanizado es un tema importante para obtener beneficios económicos en la industria. Este propósito podría alcanzarse con mayores velocidades de corte y avance. Sin embargo, el comportamiento inherente produce altas temperaturas en la interfaz de la herramienta de corte / pieza de trabajo. Muchos fluidos de corte han sido desarrollados para controlar la temperatura en el proceso y aumentar la vida de la herramienta. El objetivo de este trabajo es comparar el desgaste de la herramienta de fresado de insertos de metal duro con diferentes sistemas lubricantes/refrigerantes: Sistema Tradicional por Inundación y el Sistema de Mínima Cantidad de Lubricante (MQL). Los valores de desgaste de las herramientas de corte se evaluaron de acuerdo con la norma ISO 8688-1 1989. Los resultados experimentales mostraron que el uso del MQL reduce significativamente el desgaste de la herramienta (aproximadamente 40\%) en el fresado de acero AISI 1018 en condiciones industriales de corte.
\end{abstract}

Palabras clave: Fluidos de corte; mínima cantidad de lubricante; vida de las herramientas; fresado

\begin{abstract}
:
Nowadays, high productivity of machining is an important issue to obtain economic benefits in the industry. This purpose could be reached with high cutting velocity and feed rate. However, the inherently behavior produce high temperatures in the interface of couple cutting tool/workpiece. Many cutting fluids have been developed to control temperature in process and increase tool life. The objective of this paper is to compare the carbide milling tool wear using different systems cutting fluids: flood and minimum quantity of lubrication (MQL). The values of carbide milling cutting tool wear was evaluate according with the standard ISO 8688-1 1989. The experimental results showed that using MQL reduces significantly (about $40 \%$ ) tool wear in milling AISI 1018 steel at industrial cutting conditions.
\end{abstract}

Keywords: Cutting fluids; minimum quantity of lubrication; tool life; milling

\footnotetext{
${ }^{1}$ Universidad Técnica de Ambato, Ambato - Ecuador ( \{df.nunez, whvaca, me.urena\} @uta.edu.ec )
} 


\section{Introducción}

Hoy en día el mecanizado juega un papel importante en la industria de la fabricación mecánica. Es quizá el proceso de fabricación más versátil en el cual la forma, el tamaño y el acabado superficial deseados se consiguen mediante la eliminación del exceso de materiales en forma de pequeñas virutas. El componente que elimina el material en exceso a través de contacto mecánico directo se conoce como herramienta de corte y la máquina que proporciona el movimiento relativo necesario entre la pieza de trabajo y la herramienta de corte se denomina comúnmente máquina herramienta. Este movimiento relativo durante el mecanizado provoca una gran deformación plástica en la pieza de trabajo. Durante dicha deformación, casi el 99\% de la energía alimentada a la máquina herramienta se convierte en calor. Cuando se mecanizan materiales ferrosos y otros materiales de alta resistencia, se generan altas temperaturas en la zona de corte, lo que provoca la disminución de la resistencia, desgaste más rápido y finalmente la falla de la herramienta (Sharma, Tiwari, \& Dixit, 2016).

Muchas soluciones para conseguir un aumento en la vida de las herramientas han estado dirigidas al uso de diferentes fluidos de corte para disminuir la temperatura en la interfaz de corte pieza herramienta, (Morales, Zamora, Beltran, López K, López R, 2016) esta disminución produce menor desgaste lo que significa mayor vida útil de la herramienta de corte (Attanasio, Gelfi, Giardini, \& Remino, 2006). El uso de estos fluidos pueden producir problemas en la salud e incrementar el costo de la producción. Se reporta que los fluidos lubricantes representan entre el $7 \%$ y el $17 \%$ del costo total de producción en la industria automotriz, alcanzando hasta un $20 \%$ en aquellos materiales difíciles de mecanizar (Pereira, Rodríguez, Fernández-Abia, Barreiro, \& López de Lacalle, 2016). Solo en el 2005 se usaron 38 millones de Tm de fluidos de corte en el mundo para procesos de mecanizado, de los cuales aproximadamente el $85 \%$ son fluidos a base de petróleo. Se indica también un incremento en su utilización del 1.2\% hasta el año 2015; sin embargo, existen posibles efectos derivados de su uso como son: contaminación del aire, aguas subterráneas, suelos, agricultura, alimentos, además de su potencial irritante y alérgico (Shashidhara \& Jayaram, 2010).

Como alternativas a estos fluidos contaminantes se han realizado investigaciones con lubricantes sintéticos, sólidos y los denominados aceites vegetales. Actualmente las leyes medioambientales cada vez más restrictivas exigen una búsqueda de fluidos lubricantes con características biodegradables (Fox \& Stachowiak, 2007). En 2007 se proyectó un incremento en el uso de este tipo de fluidos del $7 \%$ al $10 \%$ en el mercado de Estados Unidos debido a que se ha demostrado que los aceites vegetales son una fuente renovable y viable como lubricantes de corte (Shashidhara \& Jayaram, 2010).

Sin embargo, los esfuerzos no solo se han encaminado a la búsqueda de fluidos de corte no contaminantes, sino también a tratar de eliminar las grandes cantidades de fluido utilizado en el 
método tradicional de mecanizado (inundación). Dentro de las nuevas técnicas de mecanizado están las de mecanizado en seco o casi seco, donde una mínima cantidad de lubricante (MQL) es esparcido justo en la interfaz de corte lo cual garantiza un buen grado de lubricación, pero el nivel de enfriamiento sigue siendo menor que el método tradicional, aunque la evacuación de la viruta se ve mejorada por el flujo de aire utilizado para pulverizar el fluido de corte (Attanasio, Gelfi, Giardini, \& Remino, 2006). Para solventar este inconveniente nuevas alternativas se han planteado; como es el mecanizado criogénico, que ha disminuido notablemente la temperatura de contacto en la zona de corte, aunque ninguna de las técnicas actuales resulta una solución efectiva cuando se trata de materiales difíciles de mecanizar muy comunes en las aplicaciones actuales de ingeniería (Shokrani, Dhokia, \& Newman, 2012).

Numerosos estudios han demostrado la efectividad del MQL en el mecanizado de materiales ferrosos usando aceites vegetales, alcoholes ésteres, lubricantes sintéticos y lubricantes sólidos a escala nanométrica, para aumentar la vida de las herramientas de corte y mejorar la calidad superficial de la pieza (Paturi, Maddu, Maruri, \& Narala, 2016) (Uysal, Demiren, \& Altan, 2015). Además se ha demostrado una reducción del 95\% del consumo de fluido de corte en relación al método tradicional por inundación y se ha resaltado la importancia de la ubicación de la boquilla dispensadora del fluido en el proceso de fresado (López de Lacalle, Angulo, Lamikiz, \& Sánchez, 2006).

En la presente investigación, mediante el uso de la norma ISO 8688-1, se compara la vida de las herramientas de corte de metal duro utilizando dos sistemas de lubricación para el proceso de fresado del acero AISI 1018, se empleó el método tradicional por inundación con aceite de corte de emulsión y el método de mínima cantidad de lubricante MQL con fluido lubricante vegetal.

\section{Materiales y Métodos}

\subsection{Máquina herramienta, pieza de trabajo y herramientas de corte}

Todos los ensayos fueron de desbaste en fresado frontal, se realizaron según la norma ISO 868811989 y se llevaron a cabo en un centro de mecanizado CNC vertical de 3 ejes (KIA V25P). El material utilizado en el fresado frontal de la pieza de trabajo fue un acero al carbono AISI 1018 de sección rectangular de 37 x $70 \mathrm{~mm}$ y una longitud de $300 \mathrm{~mm}$. La composición química y las propiedades mecánicas del acero AISI 1018 se listan en las tablas 1 y 2, respectivamente.

Tabla 1. Composición química del acero AISI 1018.

\begin{tabular}{|c|c|c|c|c|}
\hline Elemento & C & Mn & P & S \\
\hline$\% \min$. & 0.15 & 0.60 & - & - \\
\hline$\% \max$. & 0.20 & 0.90 & 0.04 & 0.05 \\
\hline
\end{tabular}

Tabla 2. Propiedades mecánicas típicas del acero AISI 1018.

\begin{tabular}{|c|c|c|c|c|}
\hline $\begin{array}{c}\text { Resistencia a la } \\
\text { tracción (MPa) }\end{array}$ & $\begin{array}{c}\text { Límite de } \\
\text { Fluencia (MPa) }\end{array}$ & $\begin{array}{c}\text { Módulo de } \\
\text { Young (GPa) }\end{array}$ & $\begin{array}{c}\text { Elongación } \\
(\%)\end{array}$ & $\begin{array}{c}\text { Dureza } \\
\text { (HB) }\end{array}$ \\
\hline 440 & 370 & 200 & 20 & 126 \\
\hline
\end{tabular}


El dispositivo portaherramientas fue de $63 \mathrm{~mm}$ de diámetro con capacidad para cinco insertos (SANDVIK / FMA01-063-A22-SE12-05). Los insertos de metal duro fueron de la serie P del tipo SANDVIK / YBG 202 SEET12T3-DR con un recubrimiento de TiAIN.

\subsection{Lubricantes y configuración experimental}

Los experimentos se llevaron a cabo usando dos sistemas de lubricación-refrigerante, el primero por inundación tradicional y el segundo por el sistema de mínima cantidad de lubricante MQL. El lubricante utilizado en el proceso de inundación fue TRICUT 2000 W/S con caudales de alimentación de $3.5 \mathrm{l} / \mathrm{min}$ y $6.5 \mathrm{l} / \mathrm{min}$ para velocidades de corte de $200 \mathrm{~m} / \mathrm{min}$ y $360 \mathrm{~m} / \mathrm{min}$, respectivamente, de acuerdo con la recomendación de la norma ISO 8688-1:1989; este es un aceite soluble con aplicaciones multipropósito ideal para el mecanizado de metales, tanto ferrosos como no ferrosos. En el MQL se trabajó con un caudal de $480 \mathrm{ml} / \mathrm{h}$ y una presión de salida de 2bars (Cervantes, 2014) (Dropsa, 2016). En este sistema se utilizó un aceite vegetal COOLUBE $2210 \mathrm{EP}$, este tipo de aceite es $100 \%$ biodegradable y no produce niebla o malos olores, ofreciendo un agradable ambiente de trabajo a los operadores. El equipo de MQL utilizado y el detalle del componente de mezclado se muestran en la Figura 1.

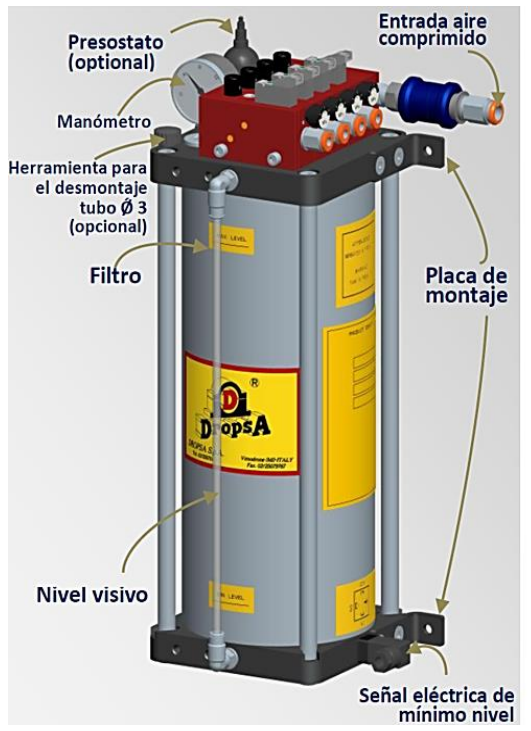

Figura 1. Equipo de MQL utilizado para el proceso de fresado frontal y el detalle del componente de mezclado aire/aceite vegetal.

Las condiciones de corte con las cuales se llevaron a cabo los experimentos se detallan en la Tabla 3.

\subsection{Medición del desgaste y determinación de la vida útil de la herramienta de corte}

El deterioro de la herramienta de corte fue evaluado acorde con la norma ISO 8688-1:1989 mediante la medición del desgaste de flanco VB (ver ilustración de la Tabla 4), donde además se estable el criterio de inutilidad de la herramienta de acuerdo con el tipo de desgaste generado en la experimentación, dicho criterio se presenta en la Tabla 4. Con los ensayos realizados se tomó el criterio de desgaste Uniforme y Normal debido a que se extiende con un ancho constante en la 
parte efectiva del filo de corte principal; por lo tanto, la inutilidad de la herramienta se presenta cuando el desgaste VB alcanza un de valor de $0.35 \mathrm{~mm}$

Tabla 3. Condiciones de corte.

\begin{tabular}{|c|c|c|}
\hline İtem & \multicolumn{2}{|c|}{ Descripción } \\
\hline Herramienta de corte & \multicolumn{2}{|c|}{$\begin{array}{l}\text { SANDVIK, Cinco insertos de metal duro : YBG } 202 \text { SEET12T3-DR } \\
\text { Recubrimiento: TiAIN } \\
\text { Portaherramienta : SANDVIK / FMA01-063-A22-SE12-05 }\end{array}$} \\
\hline Operación & \multicolumn{2}{|c|}{ Fresado Frontal } \\
\hline Velocidad de corte $(V c)$ & \multicolumn{2}{|c|}{$200 \mathrm{~m} / \mathrm{min}$ y $360 \mathrm{~m} / \mathrm{min}$} \\
\hline Avance por diente $\left(f_{z}\right)$ & \multicolumn{2}{|c|}{$0.15 \mathrm{~mm} /$ diente } \\
\hline Profundidad de pasada axial (ap) & \multicolumn{2}{|l|}{$1.2 \mathrm{~mm}$} \\
\hline Profundidad de pasada radial (ar) & \multicolumn{2}{|l|}{$37.8 \mathrm{~mm}$} \\
\hline \multirow{2}{*}{$\begin{array}{l}\text { Condiciones de enfriamiento / } \\
\text { lubricación }\end{array}$} & $\begin{array}{l}\text { Método } \\
\text { Inundación }\end{array}$ & $\begin{array}{l}\text { Aceite emulsión TRICUT } 2000 \mathrm{~W} / \mathrm{S} \\
\text { Caudal }=3.5 \mathrm{l} / \mathrm{min} \text { para } \mathrm{Vc}=200 \mathrm{~m} / \mathrm{min} \\
\text { Caudal }=6.5 \mathrm{l} / \mathrm{min} \text { para } \mathrm{Vc}=360 \mathrm{~m} / \mathrm{min}\end{array}$ \\
\hline & Método MQL & $\begin{array}{l}\text { Aceite vegetal COOLUBE 2210EP(DROPSA) } \\
\text { Caudal }=480 \mathrm{ml} / \mathrm{h} \text {; Presión de salida }=2 \text { bars }\end{array}$ \\
\hline
\end{tabular}

Tabla 4. Desgaste de flanco según la Norma ISO 8688-1:1989 y establecimiento del criterio de inutilidad.

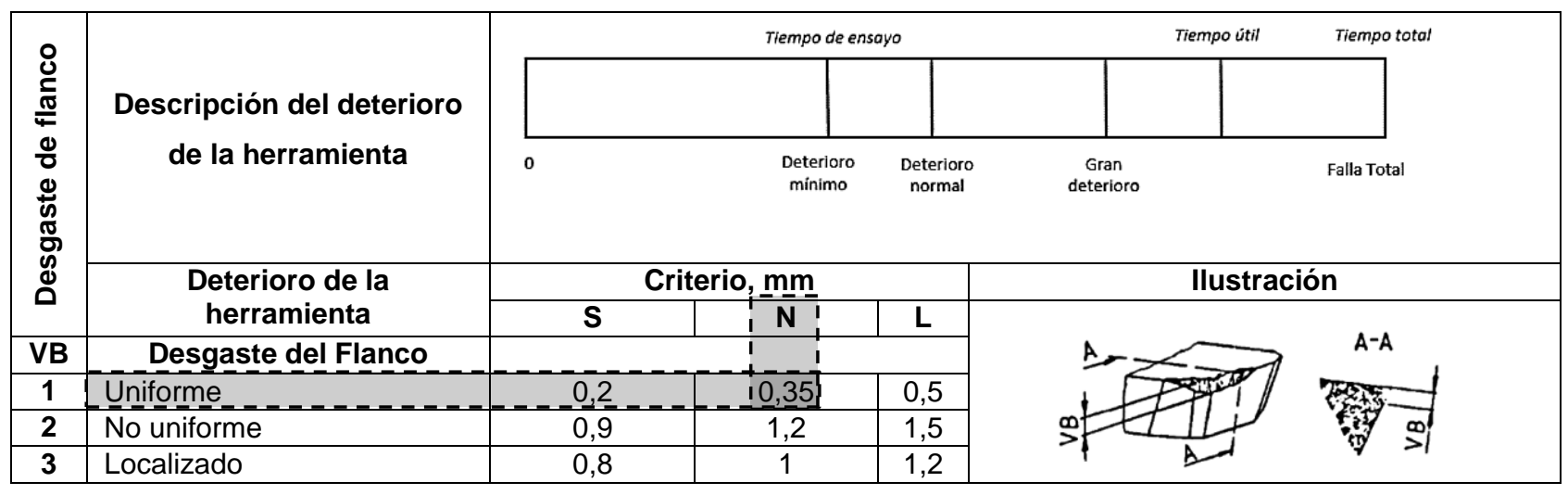

La medición precisa del valor de desgaste de flanco VB se realizó mediante un microscopio electrónico de barrido SEM (TESCAN VEGA3). El proceso de medición se realizó con interrupciones en el proceso de fresado a intervalos de 5 minutos hasta llegar al criterio de desgaste máximo $(0.35 \mathrm{~mm})$. El desgaste de flanco es el más común para determinar la vida útil de la herramienta.

\section{Resultados y Discusión}

Debido a sus muy buenas características: bajo coeficiente de desgaste, alta dureza y un comportamiento estable frente a la temperatura, el recubrimiento Nitruro de Aluminio y Titanio (Ti AIN) mejora en gran medida la vida de los insertos de metal duro.

En la Tabla 5 se muestran los resultados del desgaste de flanco VB para velocidades de corte de $360 \mathrm{~m} / \mathrm{min}$ (a) y 200m/min (b) bajo los dos métodos de lubricación: Inundación (emulsión) y mínima cantidad de lubricación (MQL). Mediante interpolación lineal se determinó que el desgaste máximo establecido por la norma ISO 8688-1:1989 de 0.35mm se alcanza para el caso del MQL a los 24.76 min.y 46,76 min, respectivamente y para el caso de inundación a los 15.38 min y a los 
33.69 min, respectivamente, lo cual indica un incremento de tiempo de vida útil de alrededor del $40 \%$ cuando se utiliza el sistema de lubricación por MQL.

En general, el espesor del recubrimiento de la herramienta de corte es muy pequeño. Si esta fina capa se deteriora la calidad del mecanizado no disminuye ya que las herramientas sin recubrimiento realizan bien su función hasta llegar al criterio de desgaste establecido. Sin embargo, cuando el recubrimiento es removido, el sustrato contacta directamente con la pieza de trabajo y el desgaste se acelera en la herramienta lo que disminuye su vida útil. (Zhang, Li, \& Wang, 2012)

Tabla 5: Valores del desgaste de flanco VB a intervalos de (a) Velocidad de corte Vc $=360(\mathrm{~m} / \mathrm{min})$ y (b) Velocidad de corte $V_{c}=200$

(a)

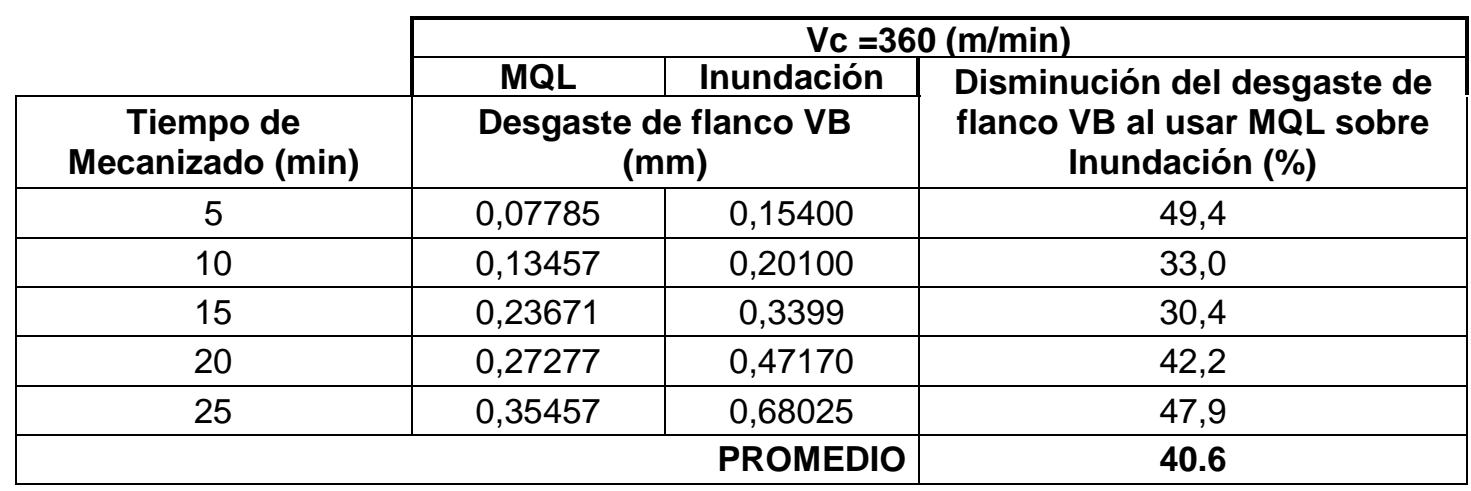

(b)

\begin{tabular}{|c|c|c|c|}
\cline { 2 - 4 } \multicolumn{1}{c|}{} & \multicolumn{3}{c|}{ Vc =200 (m/min) } \\
\cline { 2 - 4 } \multicolumn{1}{c|}{} & MQL & Inundación & $\begin{array}{c}\text { Disminución del desgaste de } \\
\text { flanco VB al usar MQL sobre } \\
\text { Inundación } \\
(\%)\end{array}$ \\
\hline $\begin{array}{c}\text { Tiempo de } \\
\begin{array}{c}\text { Mecanizado } \\
\text { (min) }\end{array}\end{array}$ & \multicolumn{2}{|c|}{$\begin{array}{c}\text { Desgaste de flanco VB } \\
(\mathbf{m m})\end{array}$} & 49,8 \\
\hline 5 & 0,05928 & 0,11798 & 45,6 \\
\hline 10 & 0,07398 & 0,13593 & 36,0 \\
\hline 15 & 0,11273 & 0,17601 & 35,2 \\
\hline 20 & 0,14047 & 0,21666 & 31,1 \\
\hline 25 & 0,16307 & 0,23677 & 35,1 \\
\hline 30 & 0,18418 & 0,28372 & 38,6 \\
\hline 35 & 0,22920 & 0,37331 & - \\
\hline 40 & 0.27507 & - & - \\
\hline 45 & 0,33230 & - & $\mathbf{3 8 , 8}$ \\
\hline 50 & 0,38268 & - & PROMEDIO \\
\hline
\end{tabular}

La Figura 2 ilustra la evolución del desgaste de flanco VB en función del tiempo de mecanizado para las velocidades de corte de $200 \mathrm{~m} / \mathrm{min}$ y $360 \mathrm{~m} / \mathrm{min}$, bajo los dos sistemas de lubricación/refrigeración, inundación Figura 2 (a) y MQL Figura 2 (b). Esta propagación del desgaste resulta una herramienta muy útil para conocer la vida de las herramientas de corte. Los 
resultados obtenidos muestran que el desgaste es significativamente afectado por las condiciones de corte, en particular por la velocidad de corte ya que se acelera para velocidades más altas, esto se presume debido al incremento de temperatura en la interfaz herramienta - pieza. Se evidencia además la presencia de tres zonas: la primera que corresponde a un desgaste preliminar, la segunda a un desgaste constante y una tercera zona, mucho más visible en el caso de inundación, donde se acelera el deterioro, conocida como zona de desgaste severo la cual corresponde a la última etapa cerca al fallo de la herramienta.

(a)

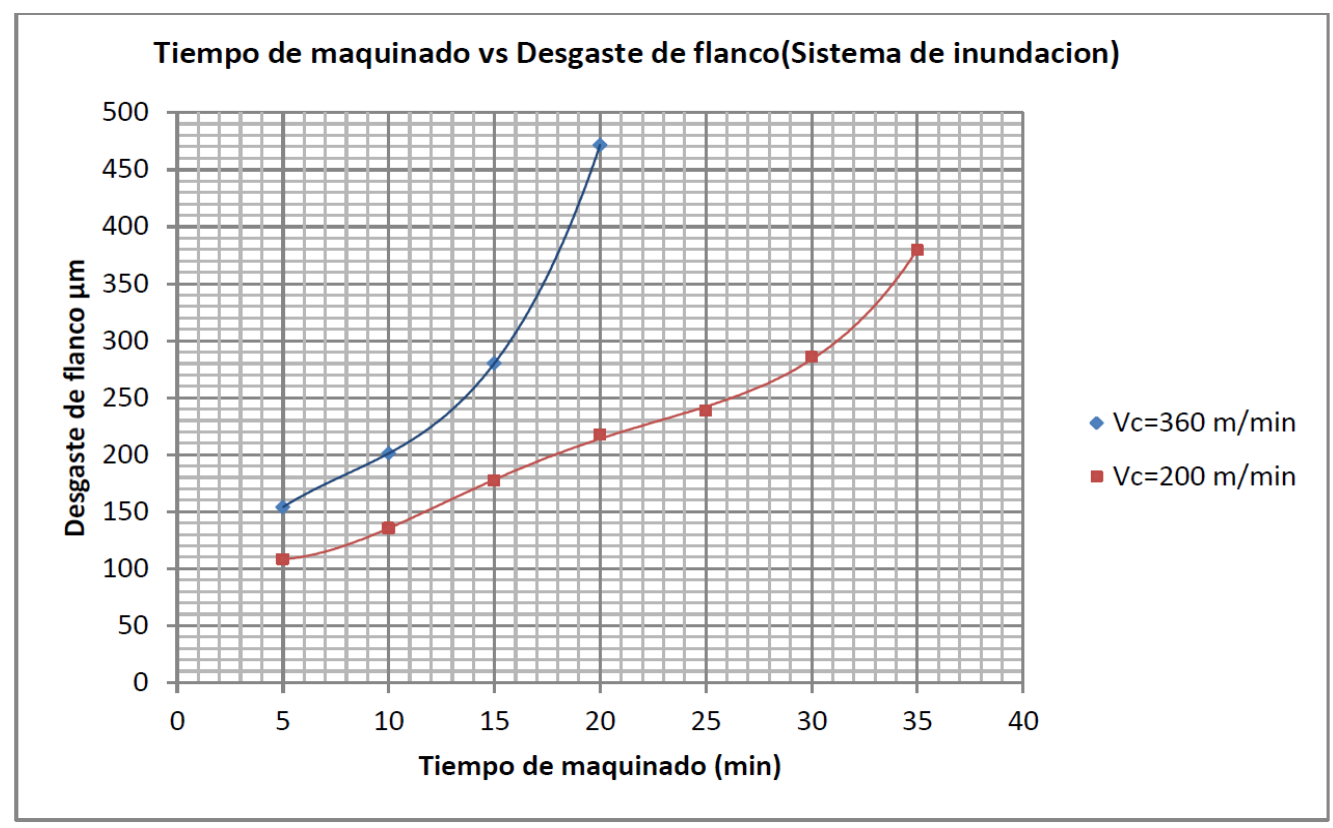

(b)

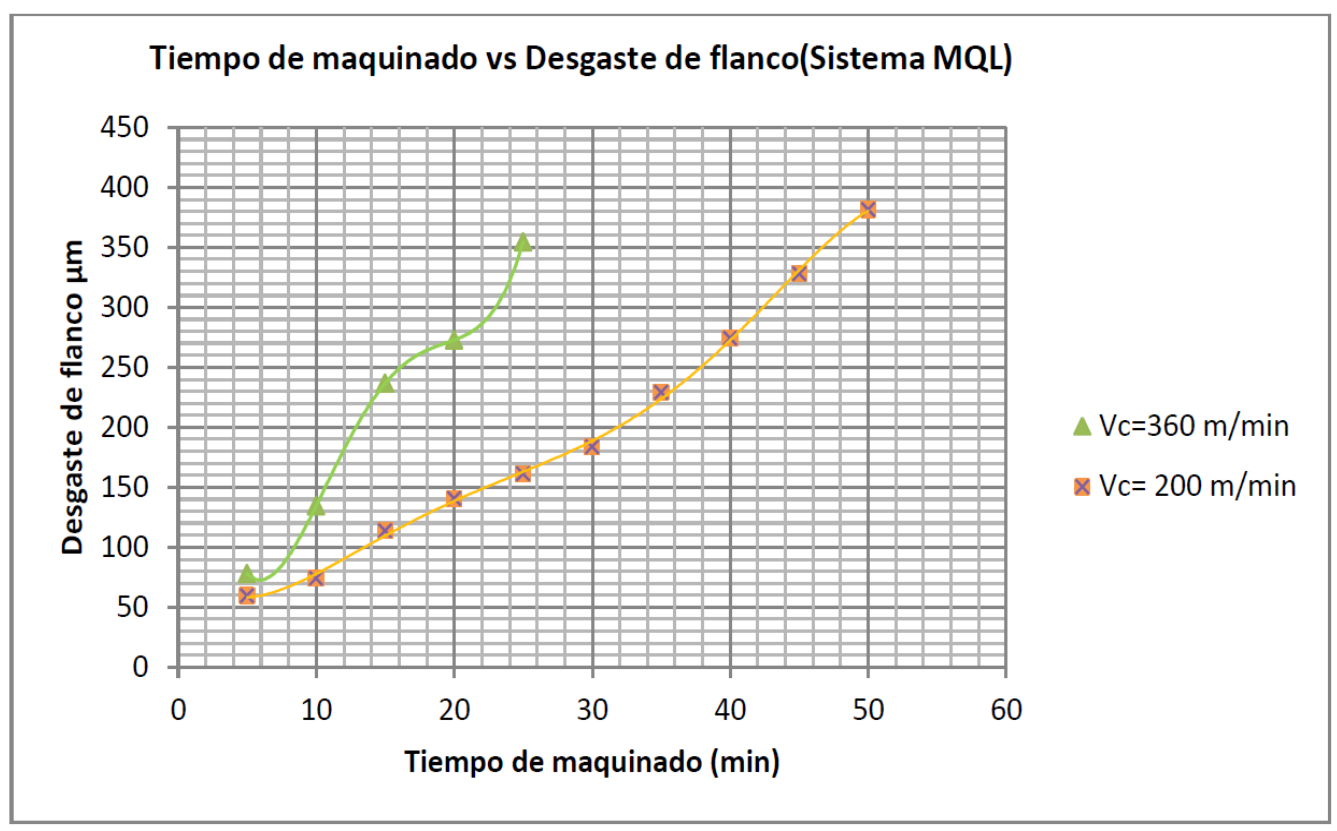

Figura 2. Evolución del desgaste de flanco en el tiempo para el caso de sistema de lubricación por: (a) inundación y (b) MQL. (Núñez, 2016) 
Una comparativa de la evolución del desgaste de flanco entre la inundación y el MQL se observa en la Figura 3 (a) para una velocidad de corte de $200 \mathrm{~m} / \mathrm{min}$ y en la Figura 3 (b) para una velocidad de corte de $360 \mathrm{~m} / \mathrm{min}$. Se muestra una disminución del desgate de flanco VB en el caso de sistema MQL lo cual implica el aumento de la vida útil de la herramienta, estos resultados son muy similares a los obtenidos por (Dhar, Kamruzzaman, \& Ahmed, 2006).

(a)

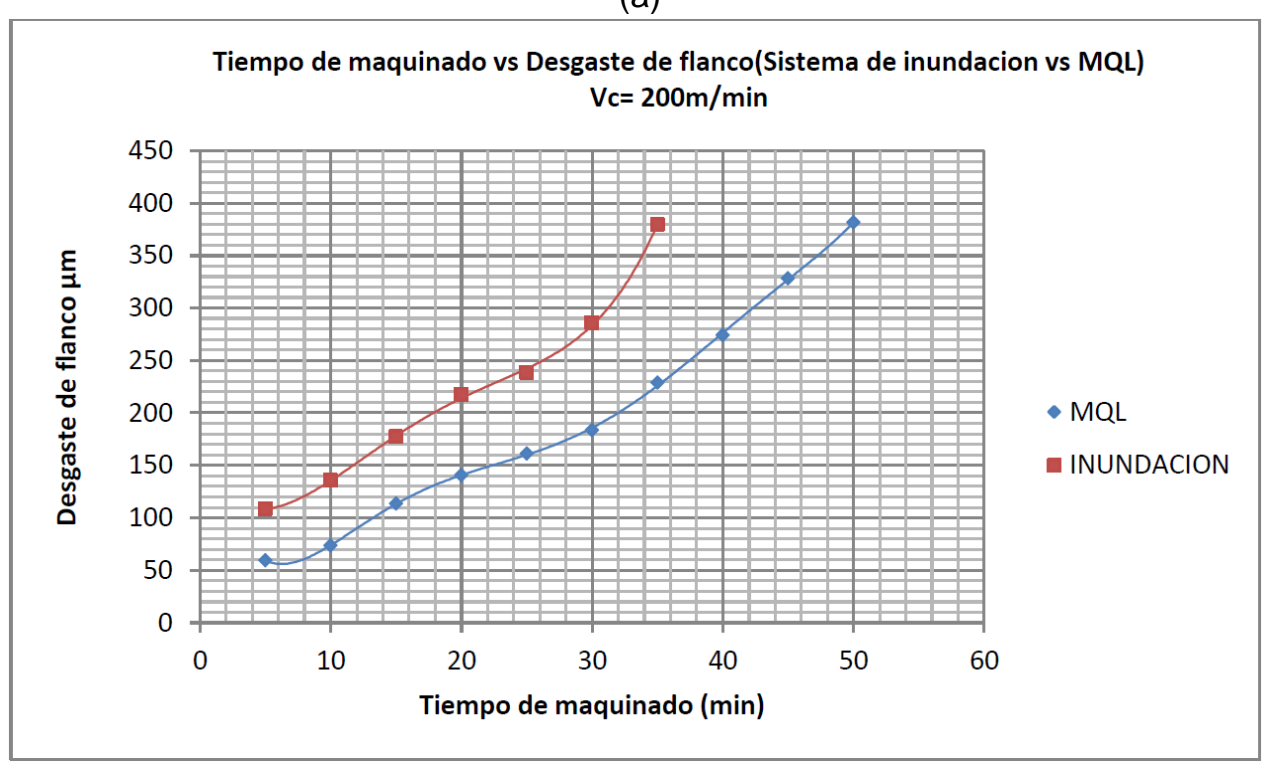

(b)

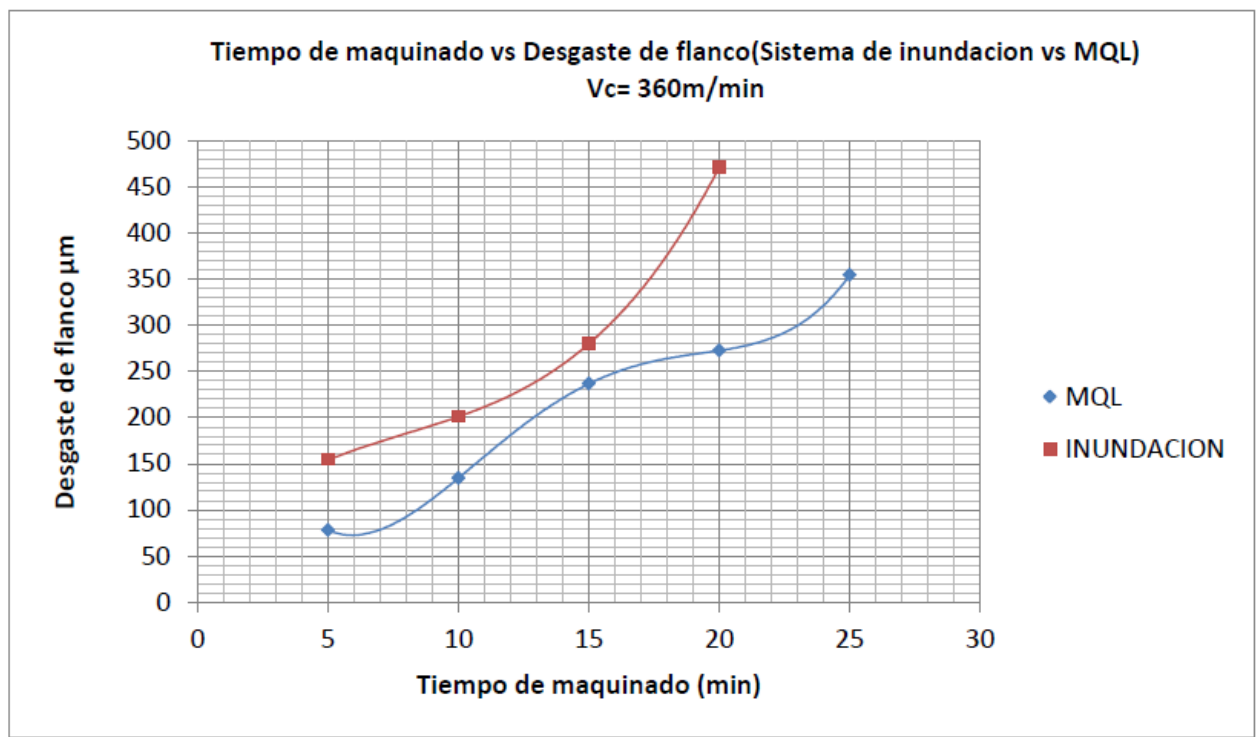

Figura 3. Comparativa del desgaste de flanco en el tiempo entre el sistema de lubricación por inundación y MQL. (a) Vc $=200 \mathrm{~m} / \mathrm{min}$ y (b) Vc=360 $\mathrm{m} / \mathrm{min}$. (Núñez, 2016)

Otro aspecto importante que diferencia al desgaste entre la inundación y el MQL se observa en la Tabla 6, donde los datos del desgaste se acompañan con las imágenes tomadas del microscopio electrónico de barrido, las mismas indican una uniformidad en el desgaste producido por el sistema de MQL mientras que para el sistema de inundación al encontrarse mayor cantidad de partículas abrasivas en la interfaz de contacto pieza / herramienta se ve un desgaste menos uniforme. Esto se presume que es debido a que la inundación no es capaz de evacuar la viruta adecuadamente de la zona de corte. 
Tabla 6: Ilustraciones del desgaste de flanco VB a Vc =360 (m/min) tomadas a intervalos de $5 \mathrm{~min}$.

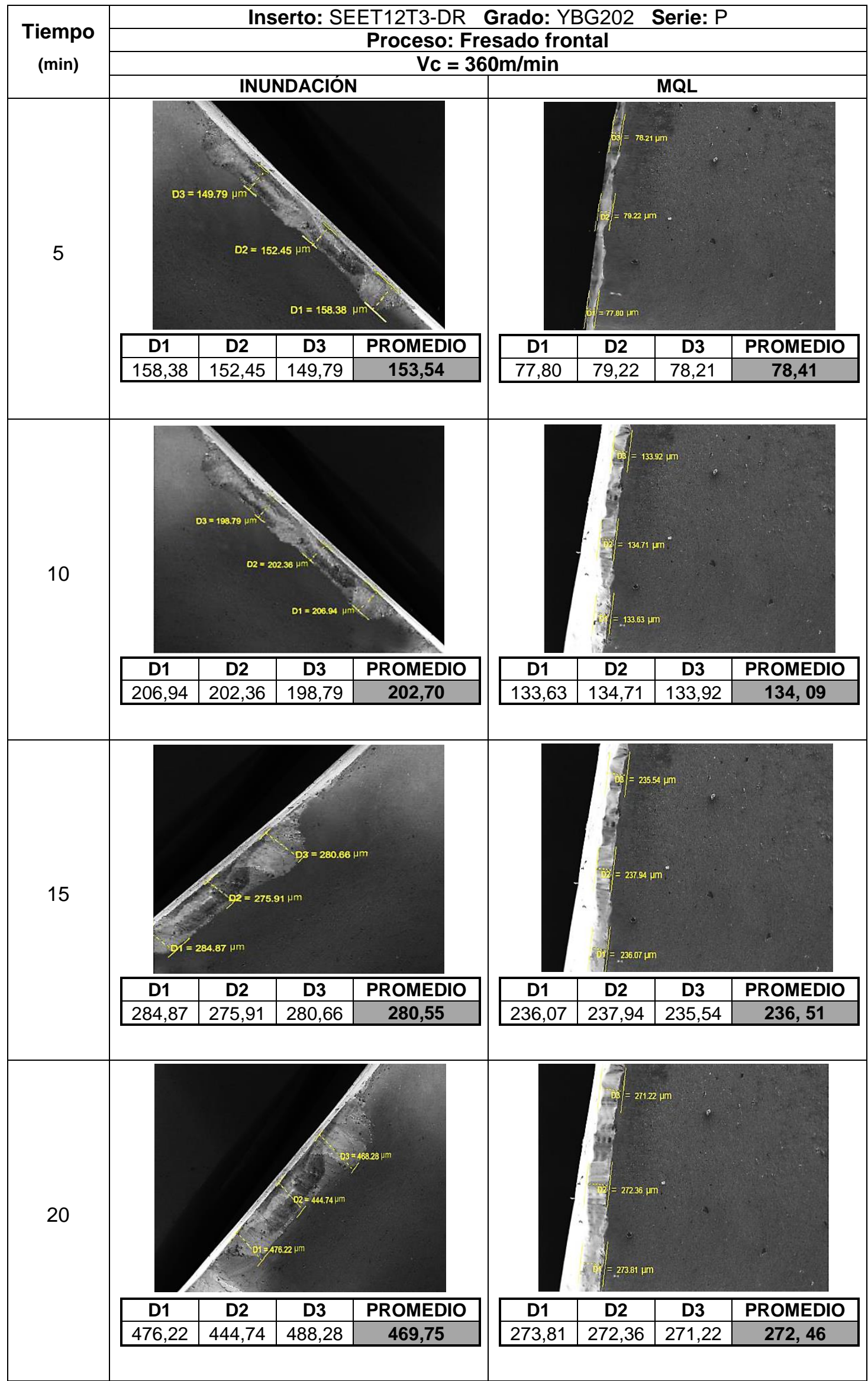




\section{Conclusiones y Recomendaciones}

La presente investigación experimental permite concluir:

- El rendimiento del sistema de lubricación - enfriamiento por Mínima Cantidad de Lubricante (MQL) aumentó en un rango del $30 \%$ al $50 \%$ respecto al sistema tradicional por Inundación debido a los siguientes beneficios: reduce el desgaste de flanco evaluado según la norma ISO 8688-1:1989 y mejora la vida de las herramientas de corte de metal duro.

- El sistema MQL reduce el consumo de herramientas de corte en los procesos productivos y puede extender los límites de velocidad de corte, además fomenta el uso de aceites amigables con el ambiente.

- El desgaste de flanco VB disminuyó en alrededor de un 40\% con el sistema MQL respecto al sistema de Inundación.

- Los resultados obtenidos muestran que el desgaste es significativamente afectado por las condiciones de corte, en particular por la velocidad de corte ya que se acelera para velocidades más altas, esto se presume debido al incremento de temperatura en la interfaz herramienta - pieza.

\section{Bibliografía}

Attanasio, A., Gelfi, M., Giardini, C., \& Remino, C. (2006). Minimal quantity lubrication in turning: Effect on tool wear. Wear, 260(3), 333-338. doi: http://dx.doi.org/10.1016/j.wear.2005.04.024

Cervantes E. (2014), MQL: Tecnologías de vanguardia de lubricación en la industria metalmecánica, Visión Industrial, Vol. 12, pp. 20-22, Recuperado de: https://issuu.com/visionindustrial/docs/vision_12_ok/3

Dhar, N. R., Kamruzzaman, M., \& Ahmed, M. (2006). Effect of minimum quantity lubrication (MQL) on tool wear and surface roughness in turning AISI-4340 steel. Journal of Materials Processing Technology, 172(2), 299-304. doi: http://dx.doi.org/10.1016/j.jmatprotec.2005.09.022

Dropsa (2016), Continuous and perfect air/oil lubrication Guarantee ted with MiQueL, Catálogo DROPSA. Recuperado de:

http://www.dropsa.com/flex/cm/pages/ServeBLOB.php/L/ES/IDPagina/842

Fox, N. J., \& Stachowiak, G. W. (2007). Vegetable oil-based lubricants-A review of oxidation. Tribology International, 40(7), 1035-1046. doi: 
http://dx.doi.org/10.1016/j.triboint.2006.10.001

López de Lacalle, L. N., Angulo, C., Lamikiz, A., \& Sánchez, J. A. (2006). Experimental and numerical investigation of the effect of spray cutting fluids in high speed milling. Journal of Materials Processing Technology, 172(1), 11-15. doi:

http://dx.doi.org/10.1016/j.jmatprotec.2005.08.014

Morales Y. Zamora Y. Beltrán R. López K. López R. (2016) Desgaste de la herramienta de corte en el torneado en seco del acero AISI 316L. Ecuador, Ingenius, 17, 36-40 doi: http://dx.doi.org/10.17163/ings.n17.2017.05

Núñez B. (2016) Estudio del efecto del sistema de Mínima Cantidad de Lubricación (MQL) con aceite vegetal en la vida de las herramientas de corte de metal duro en el fresado del acero AISI1018, Universidad Técnica de Ambato

Paturi, U. M. R., Maddu, Y. R., Maruri, R. R., \& Narala, S. K. R. (2016). Measurement and Analysis of Surface Roughness in WS2 Solid Lubricant Assisted Minimum Quantity Lubrication (MQL) Turning of Inconel 718. Procedia CIRP, 40, 138-143. doi:

http://dx.doi.org/10.1016/j.procir.2016.01.082

Pereira, O., Rodríguez, A., Fernández-Abia, A. I., Barreiro, J., \& López de Lacalle, L. N. (2016). Cryogenic and minimum quantity lubrication for an eco-efficiency turning of AISI 304. Journal of Cleaner Production, 139, 440-449. doi:

http://dx.doi.org/10.1016/j.jclepro.2016.08.030

Sharma, A. K., Tiwari, A. K., \& Dixit, A. R. (2016). Effects of Minimum Quantity Lubrication (MQL) in machining processes using conventional and nanofluid based cutting fluids: A comprehensive review. Journal of Cleaner Production, 127, 1-18. doi: http://dx.doi.org/10.1016/j.jclepro.2016.03.146

Shashidhara, Y. M., \& Jayaram, S. R. (2010). Vegetable oils as a potential cutting fluid-An evolution. Tribology International, 43(5-6), 1073-1081. doi:

http://dx.doi.org/10.1016/j.triboint.2009.12.065

Shokrani, A., Dhokia, V., \& Newman, S. T. (2012). Environmentally conscious machining of difficult-to-machine materials with regard to cutting fluids. International Journal of Machine Tools and Manufacture, 57, 83-101. doi:

http://dx.doi.org/10.1016/j.jjmachtools.2012.02.002

Uysal, A., Demiren, F., \& Altan, E. (2015). Applying Minimum Quantity Lubrication (MQL) Method on Milling of Martensitic Stainless Steel by Using Nano Mos2 Reinforced Vegetable Cutting Fluid. Procedia - Social and Behavioral Sciences, 195, 2742-2747. doi: 
http://dx.doi.org/10.1016/j.sbspro.2015.06.384

Zhang, S., Li, J. F., \& Wang, Y. W. (2012). Tool life and cutting forces in end milling Inconel 718 under dry and minimum quantity cooling lubrication cutting conditions. Journal of Cleaner Production, 32, 81-87. doi:

http://dx.doi.org/10.1016/j.jclepro.2012.03.014 